\title{
El método científico y la filosofía como herramientas para generar conocimiento
}

Sandra Milena De Hoyos Benítez

Corporación Unificada Nacional de Educación

Superior, Colombia 


\title{
El método científico y la filosofía como herramientas para generar conocimiento*
}

\begin{abstract}
Resumen: desde tiempos inmemorables el hombre ha cuestionado el mundo que lo rodea y el porqué de los fenómenos que diariamente observa en la naturaleza, al igual que se ha interesado por estudiar el comportamiento humano y la explicación de las interacciones humanas. En la búsqueda por explicar lo que le rodea, el hombre ha propuesto hipótesis que con el paso del tiempo se han podido verificar o falsear, ese conjunto de conocimientos que se ha logrado confirmar o refutar es lo que se conoce como ciencia y el conjunto de pasos que se siguen para poder confirmarlos es lo que se conoce como método científico. Este artículo busca hacer un análisis de las distintas definiciones de ciencia que se han conocido provenientes de pensadores y filósofos a lo largo de la historia; se realiza un análisis de la categorización de métodos científicos aplicados para la generación de conocimiento y cómo la filosofía aporta para la generación de este.
\end{abstract}

Palabras clave: ciencia, método, paradigma, conocimiento, verdad.

\section{The scientific method and philosophy as tools for knowledge generation}

\begin{abstract}
: since immemorial times, the man has questioned the world around him and the reasons of the phenomena he observes in nature daily. He has also interested in studying human behavior and explaining human interactions. In the search of explaining what is around him, the man has proposed hypotheses that with the pass of time have been verified or distorted; that body of knowledge which have been able to be confirmed or refuted is what is known as science and the group of stages that are followed to confirm knowledge is what is known as scientific method. This article is aimed to make an analysis of the different definitions of science that have come from thinkers and philosophers along the history and also provides an analysis of the categorization of scientific methods that are applied for the generation of knowledge and how philosophy contributes to its generation.
\end{abstract}

Keywords: science, method, paradigm, knowledge, truth.

Fecha de recepción: 21 de enero de 2019

Fecha de aceptación: 15 de mayo de 2019

Forma de citar (APA): DeHoyos-Benítez, S. (2020). El método científico y la filosofía como herramientas para generar conocimiento. Revista Filosofía UIS, 19(1), DOI: 10.18273/revfil.v19n1-2020010

Forma de citar (Harvard): DeHoyos-Benítez, S. (2020). El método científico y la filosofía como herramientas para generar conocimiento. Revista Filosofía UIS, 19(1), 229-245.

Sandra Milena De Hoyos Benítez: colombiana. Magíster en gestión de la innovación. Profesora en la Corporación Unificada de Educación Superior, Colombia.

ORCID iD: orcid.org/0000-0001-7192-5332

Correo electrónico: sandra_hoyos@cun.edu.co

*Artículo de reflexión derivado de investigación. 


\section{El método científico y la filosofía como herramientas para generar conocimiento}

\section{Introducción}

Durante años, la filosofía de la ciencia se ha dedicado a entender el significado de la ciencia y cómo esta se diferencia de otras actividades diarias del hombre que aportan al desarrollo de la humanidad. A través de la ciencia, el hombre ha logrado adquirir, de manera sistemática, conocimientos sobre el mundo que lo rodea, sobre la naturaleza en sí misma y los seres que interactúan en esa naturaleza, ha podido comprender los fenómenos y las leyes que rigen estos fenómenos que ocurren en el mundo, pero, principalmente, la ciencia ha permitido que el hombre deje de lado la incertidumbre que genera el desconocimiento de su mundo.

Inicialmente, el hombre se hace preguntas sobre ciertos acontecimientos sobre los cuales aún se desconoce su explicación. De manera provisional, el hombre hace un planteamiento a partir del cual se hace un esfuerzo y una búsqueda por encontrar la explicación de esos fenómenos, es así como, en la búsqueda para entender el mundo, el hombre aplica (en algunos casos sin saberlo) el método científico.

A continuación, se hace una recopilación sobre las distintas definiciones del término ciencia, se hace un análisis acerca de las etapas que conforman el método científico y las distintas acepciones de algunos filósofos representativos en cuanto a las distintas formas de aplicación de dicho método. De igual forma, se explica el aporte que hace la filosofía para la generación del conocimiento desde la perspectiva de la filosofía de la ciencia.

\section{El concepto de ciencia}

Son muchos los filósofos y pensadores que han dado su definición de ciencia y han aportado al enriquecimiento de este concepto. Aristóteles, por ejemplo, estableció la diferencia entre ciencia y experiencia: para él, la ciencia es el conocimiento de lo universal, que es demostrable y válido para todos; mientras que la experiencia es el conocimiento de lo particular, circunstancial, para 
casos específicos de una determinada realidad. El instrumento del que deben valerse todas las ciencias, según Aristóteles, es la lógica y, a su vez, establece que la observación es la base de todas las ciencias. En la obra Segundos analíticos, Aristóteles (1995) habla de las necesidades específicas de la demostración, así como de la definición y el conocimiento científico. Según Mié, la ciencia aristotélica consiste en:

[...] Un conjunto limitado de proposiciones básicas (axiomas, hipótesis y definiciones), restringidas por su pertenencia al mismo género, y articuladas en principios y teoremas demostrados, los cuales se componen de conceptos organizados en un orden de dependencia unidireccional y no reversible, desde los superiores y primeros hasta los inferiores y derivados (2009, p. 4).

A su vez, Aristóteles clasificó las ciencias en teoréticas, prácticas y productivas. Bajo esta clasificación, las ciencias teoréticas son aquellas que tienen como objetivo alcanzar el conocimiento teórico de la realidad, por ejemplo, la física, la biología o la matemática; las ciencias categorizadas como teoréticas son también conocidas como ciencias superiores dado que investigan las leyes de la realidad. En lo referente a las ciencias prácticas, estas se ocupan de las conductas, la acción y el comportamiento humano, ejemplos de estas ciencias son la ética, la política o el derecho. En cuanto a las ciencias productivas o técnicas, estas son las orientadas al hacer y se basan en la producción, como la medicina o la ingeniería.

Desde el punto de vista del objeto de estudio, las ciencias se dividen en fácticas y formales: las fácticas — como las ciencias naturales y sociales- utilizan la investigación para descubrir cómo funcionan los fenómenos observados, mientras que las formales — como la lógica y la matemática - se ocupan de inventar entes formales y de establecer relaciones entre ellos (Bunge, 2013). Es así como la lógica y la matemática se concentran en entes que solo existen en la mente mediante la abstracción de objetos reales, por lo tanto, su materia prima no es real y palpable. Por otro lado, las ciencias formales proponen enunciados que relacionan signos, mientras que los enunciados de las ciencias fácticas se concentran en sucesos o procesos. Incluso, una vez propuesto el enunciado, sigue existiendo una diferencia en lo concerniente al método por medio el cual se verifica este: mientras las ciencias formales utilizan la lógica para verificar, las fácticas utilizan la observación y experimentación.

Ahora, centraremos la atención en las características que comparten las ciencias fácticas: estas, por ser provenientes de la filosofía, se enriquecen de la matemática y la lógica, no como herramientas sino como disciplinas con las cuales los científicos aprenden a pensar rigurosamente sobre el mundo empírico; sin embargo, contrario a las ciencias formales, las ciencias fácticas se basan en suposiciones a posteriori, teorías e hipótesis. Cuando nos detenemos a mirar el mundo en el que vivimos, la mente ya tiene una teoría sobre cómo interpretar 
lo que percibe y cuando las ciencias fácticas confrontan esa realidad lo hacen basándose en teorías preexistentes.

En concordancia, los científicos parten de una realidad palpable y observable y la perturban de tal manera que obtienen datos empíricos que podrán utilizar para elaborar una teoría científica. Pareciera, entonces, que los científicos no se conformaran con la realidad que está ante sus ojos, sino que siempre la cuestionan e intervienen para producir nuevos hechos y sus explicaciones, tal como lo hace un filósofo, razón suficiente para que autores como More (2012) afirmen que la evidencia es el núcleo del método científico, que proporciona la base para adquirir nuevos y corregir y consolidar el conocimiento.

Asimismo, la ciencia no analiza la realidad como un todo, sino que busca dividir esa realidad en pequeñas partes que permitan entender el mecanismo por el cual se presenta dicha realidad a través de la aplicación de un método científico cuyo resultado final es un conocimiento preciso y claro, pero no necesariamente irrefutable; es decir, el conocimiento científico resultante debe ser publicado a fin de que pueda ser puesto en materia de juicio por otros científicos y pueda llegar a ser confirmado o refutado. Luego, tras haber verificado las hipótesis, el conocimiento científico explica los hechos observados en términos de leyes, pero no se queda solo en la explicación, de ahí que los científicos actúan como filósofos al procurar responder por qué ocurren los hechos de la forma que ocurren y no de otra manera. A pesar de la precisión y la claridad, algunas hipótesis planteadas por las ciencias pueden estar erradas, no solo por estar mal formuladas sino por lo que suponen en sí, es decir, por inexactitud de los enunciados de las leyes o por imprecisión de la información disponible (Bunge, 2014), de manera que al principio vemos todo a través de los ojos de la credibilidad hasta que aparece el filósofo para empezar a ver el mundo de una manera diferente desarrollando todas las teorías y paradigmas a través de la historia.

Hasta ahora, se ha podido reafirmar que la ciencia es empírica y permite describir los hechos reales de forma detallada haciendo uso del método científico pues este permite obtener resultados lógicos y comprobables. Además, la ciencia también se considera abierta ya que no hay límites para el desarrollo del conocimiento y puede trascender por diferentes generaciones. En ese sentido, Gárnica afirma que:

El aporte de datos objetivos para la obtención de información es la mayor utilidad para generar conocimiento [...] una de las finalidades básicas de la ciencia es conseguir explicar por qué se producen los hechos, y demostrar de qué manera ocurren (2016, p. 109).

Según lo anterior, la epistemología y la filosofía de la ciencia han realizado grandes aportes a la ciencia porque indagan y buscan la verdad basándonos en 
hipótesis que son verificadas a través de un método científico que nos permite ampliar el conocimiento. Popper (1972) tuvo otra visión de la ciencia, entendida esta como un sistema de enunciado absoluto y verdadero o teorías comprobadas; la denominó la concepción autoritaria del conocimiento científico. Popper sostuvo que el avance de la ciencia no se da debido a la acumulación de más experiencias perceptivas derivadas con el paso del tiempo, ni tampoco por la utilización de las percepciones y sentidos; por el contrario, solo se puede entender a la naturaleza por medio de teorías audaces, que son herramientas para captarla (Chávez, Hernández, Chávez y Espinosa, 2016). Fue así que Popper estableció la diferencia entre ciencia y seudo-ciencia, ya que afirmaba que la seudo-ciencia consistía en aquellos métodos de búsqueda de conocimiento que solo buscan confirmar creencias, un ejemplo de esto es la creencia de Santa Claus, es decir, el mundo está lleno de imágenes y objetos que "evidencian" la existencia de Santa Claus pero, según él, solo si se busca refutar su existencia se llegaría a demostrar su inexistencia.

De esta manera, si se quiere probar una teoría es necesario formular la siguiente pregunta: ¿se busca confirmarla o refutarla? A partir de esta pregunta surge la diferencia entre la ciencia y la seudo-ciencia, pues, según Popper, la ciencia busca refutar, mientras la seudo-ciencia busca confirmar. Popper basó su aseveración en que es fácil confirmar una teoría si se busca su confirmación y que dicha confirmación solo contaba si provenía de predicciones riesgosas, es decir, aquellas predicciones que podrían llegar a destruir la teoría. En otras palabras, para Popper cada creencia falsa que se encuentre es buena en el sentido en que nos lleva más a creer solo verdades, de ahí que para él la única forma genuina de confirmar una teoría era buscando refutarla y que se esmerara tanto por hacer entender al mundo que aquellas teorías que son irrefutables no son científicas. Se concluye entonces que no es necesario probar una hipótesis científica, sino que basta con poder refutarla; en palabras de Popper significa que "una teoría es científica si, y solo si, sus proponentes pueden especificar a priori qué hallazgos la refutarían" (Popper, citado en Herbert et al., 2000). Así pues, el concepto de ciencia y seudo-ciencia de Popper está muy permeado por la filosofía en el sentido en el que se debe estar abierto a la idea de que una creencia sea falsa porque es la única manera en que, aferrándonos a ella, pueda llegar a tener algún significado.

Por su parte, Kuhn (2002) diferenciaba entre dos tipos de ciencia: la ciencia normal y ciencia revolucionaria o extraordinaria. En ambos tipos de ciencia lo que lleva al progreso es la resolución de problemas; durante la ciencia normal, la necesidad de resolver un problema se satisface a través de un paradigma; durante la ciencia extraordinaria la necesidad de resolver el problema perdura, pero ahora se satisface encontrando un paradigma que lo reemplace, de hecho, ambas ciencias corresponden a fases de lo que se conoce como el ciclo de Kuhn explicado en la obra The Structure of Scientific Revolutions, en el año 1962. 
Hasta este momento se ha mencionado el término paradigma en un par de ocasiones, pero ¿qué es un paradigma?, Khun afirma que el término paradigma se usa de dos sentidos diferentes: por un lado, representa toda la constelación de creencias, valores, técnicas, y demás, compartidos por los miembros de una comunidad determinada (Khun, 1970; citado por Hassan \& Mingers, 2018). En el otro, denota un conjunto concreto de elementos de esa constelación que se emplean como modelos o ejemplos, y que pueden reemplazar las reglas explícitas como base para la solución de los cuestionamientos de la ciencia normal. Además, Kuhn habla sobre una pre-ciencia también llamada estado de pre-paradigma, en esta pre-ciencia las personas describen y explican de diversas maneras un determinado fenómeno, es decir, cada persona que trata de explicar ese fenómeno defiende y se aferra a su punto de vista y no existe un consenso básico sobre el tema de estudio. A partir de esta pre-ciencia se inicia el ciclo de Kuhn que se muestra en la figura 1.

Figura 1. Ciclo de Kuhn

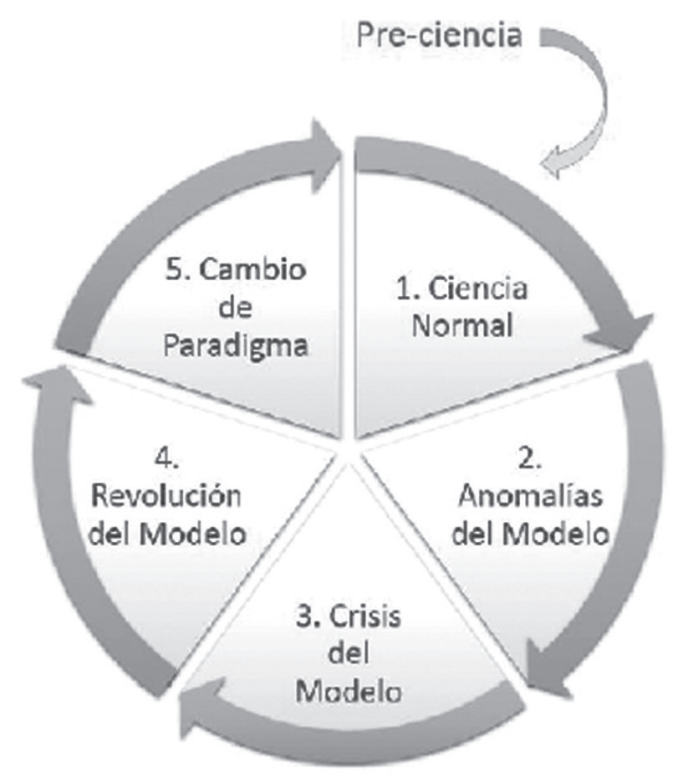

Fuente: elaboración propia

El ciclo de Kuhn inicia entonces con la llamada ciencia normal. Para Kuhn, la ciencia normal es aquella actividad de investigación guiada por un paradigma. En el campo de las humanidades, realizar investigaciones sobre el comportamiento humano suele requerir la adopción de un paradigma con el objetivo de mejorar la credibilidad y la generalización del estudio, sin embargo, la aplicación de paradigmas en la investigación varía de un investigador a otro ya sea por la 
naturaleza del fenómeno que se estudia o por elección del investigador (Kankam, 2019). Los resultados que se obtienen a partir de ese proceso de investigación aumentan la precisión del paradigma, pero no producen novedades significativas.

La segunda fase del ciclo, anomalías del modelo, se da cuando se presentan problemas que no pueden ser resueltos con las herramientas conceptuales del paradigma, esta fase se presta para que se piense que algo anda mal en el paradigma propuesto y que solo se puede llegar a solucionar dicho problema si se hacen cambios en los supuestos básicos planteados; en otras palabras, en esta etapa se pone en duda la eficacia del paradigma vigente. La presencia de anomalías conlleva la etapa de crisis, en esta fase se da la llamada ciencia extraordinaria o revolucionaria anteriormente mencionada: aquí se proponen alternativas a las estructuras teóricas previamente existentes que necesariamente implican un rechazo a los supuestos que hasta el momento se habían aceptado.

La fase de crisis llega a su fin cuando el paradigma que fue puesto en tela de juicio logra demostrar que puede resolver los problemas que precisamente provocaron la crisis o cuando aparece un nuevo enfoque que puede ser adoptado como paradigma y que llevará a que empiecen las luchas para tratar de llegar a un consenso, la aparición de ese nuevo paradigma es lo que se conoce como revolución del modelo. Después de un período en el que compiten diversos puntos de vista, ciertas teorías comienzan a atraer adeptos y se forman escuelas de pensamiento, gradualmente, esta fase da paso a una siguiente etapa de desarrollo en la que una escuela "gana estatus" (Schwartz, 2018). Una vez que se llega a un consenso y se adopta por la comunidad científica el nuevo paradigma entonces se llega a la etapa de cambio de paradigma.

Desde otro punto de vista, Kant (1998) indagó sobre las condiciones que hacen posible el conocimiento científico y encontró dos condiciones: las empíricas y las a priori. Las condiciones empíricas provienen de los sentidos, es decir, son percibidas por nuestros sentidos, son particulares y puede que se den o no; en cambio, las condiciones a priori son universales y necesarias, se dan antes de las condiciones empíricas y pertenecen a la estructura del sujeto. Adicionalmente, Kant afirmaba que la ciencia se expresa mediante juicios, pudiendo estos ser de tipo analítico o sintético. En ese sentido, los juicios analíticos no aportan al conocimiento, ya que no dicen nada que no esté ya en el sujeto, mientras que los juicios sintéticos sí amplían nuestro conocimiento. Pero Kant, también proponía otra categorización de los juicios: los juicios a priori o juicios a posteriori. Los juicios a priori no se basan en la experiencia o en la percepción del mundo, en cambio para el caso de los juicios a posteriori, se debe acudir a la experiencia para saber si son ciertos. Entonces, todo juicio analítico es a priori y todo juicio sintético es a posteriori. En concordancia con lo que afirma Kant, Falkenburg (2018) llega a la conclusión de que el análisis debe preceder a la síntesis y que el punto de 
inicio del análisis deben ser los fenómenos en lugar de los conceptos metafísicos arbitrarios.

\section{El método científico}

A las personas que se dedicaban a la ciencia se les conoció como hombres de ciencia o filósofos de la naturaleza, hasta que el filósofo y teólogo William Whewell acuñó el término científico, tal como lo refiere Whewell:

We need very much a name to describe a cultivator of science in general. I should incline to call him a Scientist. Thus we might say, that as an Artist is a Musician, Painter, or Poet, a Scientist is a Mathematician, Physicist, or Naturalist (1840, p. cxiii).

Una vez se tuvo el concepto científico, se empezó a identificar que estos pensadores seguían una metodología o proceso para obtener nuevos conocimientos y que este proceso tenía como finalidad el establecimiento de relaciones entre hechos, para enunciar leyes que explicaran el funcionamiento del mundo, esto es lo que se conoce como método científico.

A menudo se considera que el filósofo griego Tales de Mileto es el creador del método científico puesto que él, en lugar de confiar en una explicación sobrenatural de los fenómenos, buscó explicaciones naturalistas de lo que observaba. Tales de Mileto creyó que existía una sustancia primaria a partir de la cual todo lo demás se origina y llegó a la conclusión de que esa sustancia era el agua. Entonces, el problema consistió en explicar cómo todo se originaba a partir de este elemento. La respuesta fue que la tierra flotaba sobre un océano cósmico, y la hipótesis, que la tierra se comportaba flotando sobre el agua, como un pedazo de madera; Tales llegó a esta hipótesis porque vivía en una ciudad costera y a menudo veía cómo barcos muy pesados eran capaces de flotar siendo "más pesados que el agua". Esta historia demuestra que las ideas de Tales de Mileto surgieron de lo que él observaba y cómo, a partir de esto planteaba hipótesis para intentar explicar los fenómenos observados.

Doscientos años más tarde, Aristóteles hizo incontables observaciones de la naturaleza, especialmente en lo que tiene que ver con atributos de las plantas y hábitos de los animales, de igual forma hizo muchas observaciones sobre el funcionamiento del universo y de allí se originó una teoría del universo. Aristóteles trabajaba de tal forma que se hacía preguntas y planteaba respuestas para llegar a "verdades" o axiomas, al tiempo que desarrollaba "leyes de razonamiento" para poder explicar sus observaciones.

Luego, empezó la llamada Revolución Científica en cabeza de pensadores como Bacon, Newton y Galileo. El trabajo de Bacon no es tan reconocido por su 
labor experimental sino por promover la ciencia desde la óptica de la recopilación de datos, para luego, a través del razonamiento deductivo, llegar a conclusiones que explicaran los fenómenos. Por otro lado, Popper defendía el sesgo de la ciencia al afirmar que esta avanza por medio de la falsificación en la medida en que, si se puede demostrar que una teoría es falsable, entonces es científica, si no puede, entonces es seudo-ciencia y, por su parte, Kuhn expuso que los científicos generan una serie de puntos de vista teóricos a partir de los cuales adoptan uno que llega a ser paradigma hasta que otro lo reemplace. Hasta aquí, todos los pensadores mencionados hicieron su valioso aporte a lo que se conoce como método científico.

Como toda técnica, el método científico está compuesto de unos pasos secuenciales para llevarse a cabo (ver figura 2). La primera etapa tiene que ver con identificar el problema a abordar; este problema puede darse por la ausencia de conocimiento, por una pregunta que necesita una respuesta o por la necesidad de explicar datos preexistentes. En la segunda etapa, se propone una hipótesis con el objetivo de buscar una solución provisional al problema que se plantea. En la tercera etapa se recurre a la experimentación u observación para comprobar las hipótesis planteadas; en esta etapa se realiza la recogida, análisis e interpretación de los datos (Asensi y Parra, 2002, p. 6). En la cuarta etapa se realiza la verificación de los resultados obtenidos y, por último, en la quinta etapa se realiza el análisis de los resultados y el diseño del nuevo esquema mental.

Figura 2. Etapas del método científico

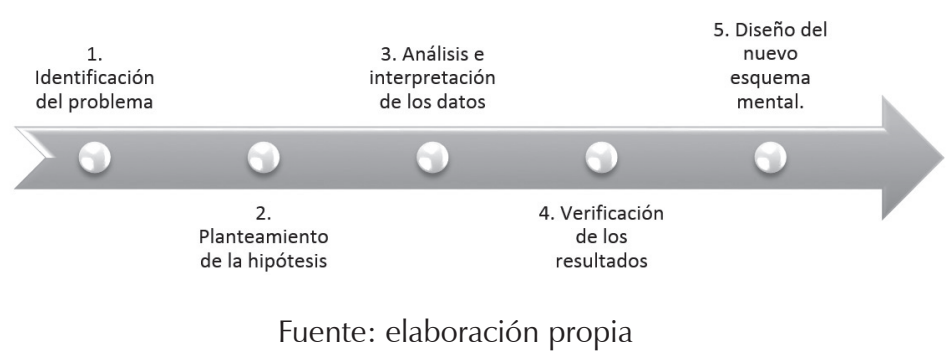

A través de la historia de la filosofía de la ciencia se pueden destacar varias categorías del método científico: la primera categoría corresponde al método inductivo-deductivo. El método inductivo-deductivo fue defendido por filósofos como Aristóteles, Francis Bacon y otros positivistas lógicos. Aristóteles, por ejemplo, en el libro Los Segundos Analíticos analiza diversos problemas relacionados con el enfoque científico y expone que todo saber proviene de uno anterior, de un conocimiento preexistente. Así mismo, Aristóteles consideró que la investigación científica va desde una observación primaria hasta llegar a lo general y que el 
científico, a través de los fenómenos que ha observado debe inducir principios explicativos para luego deducir enunciados acerca de estos fenómenos. Es decir, primeramente, se deben crear teorías que expliquen los fenómenos observados, y luego se deben deducir leyes generales para dichos fenómenos. Por su parte, Bacon propuso que el investigador primero debe establecer conclusiones generales, basándose en hechos recopilados mediante la observación directa, y aconsejó observar a la naturaleza directamente, reunir datos particulares y hacer generalizaciones a partir de ellos (Dávila, 2006).

La segunda categoría de método científico corresponde al método a priorideductivo. Entre los pensadores que han militado en este grupo se encuentran Pitágoras, Platón, Berkeley, Kant y la mayor parte de los racionalistas. Según estos pensadores, se llega al conocimiento científico a partir de una serie de principios generales, de los cuales se deducen instancias particulares que pueden o no ser demostradas objetivamente. En ese orden de ideas, dentro del arco del conocimiento de Platón el desarrollo del conocimiento procede de la inducción a la deducción, es decir, las percepciones sensoriales proporcionan la base para llegar a las teorías universales, las cuales a su vez están respaldadas por explicaciones comprobables. El mencionado arco del conocimiento de Platón se explica en la figura 3 adaptada de Sale \& Thielke (2018).

Figura 3. El arco del conocimiento

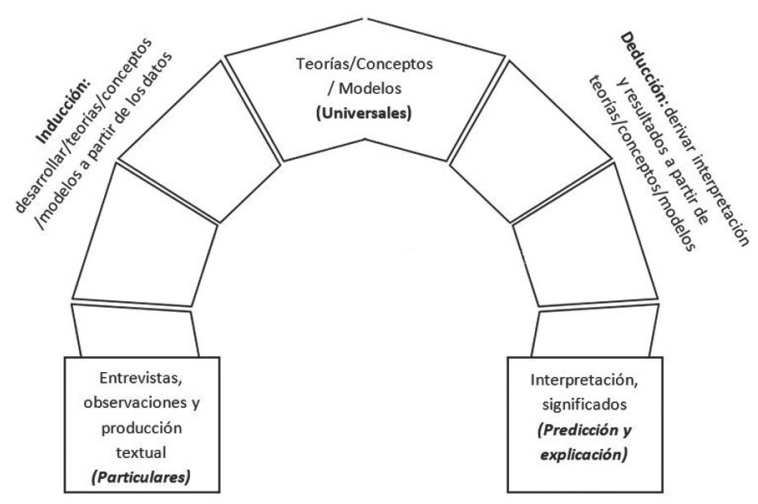

Fuente: Sale \& Thielke

A partir de la analogía de un arco arquitectónico, Platón se refirió a la inducción como el medio para ascender de los detalles a los universales. Con detalles se refería a las percepciones sensoriales captadas a través de hechos observables (entrevistas, observación y producción textual) o resultados experimentales. Los universales corresponden entonces a las leyes, principios o teorías. El descenso del arco implica el proceso lógico que permite generar predicciones y explicaciones 
de los universales, es decir, la deducción. Así pues, la ciencia no es simplemente el conjunto de universales en el vértice del arco, sino el arco en sí.

De igual forma, Platón, en su teoría de las ideas, propone que las ideas son entes universales, perfectos y con existencia verdadera (objetiva), de las que los hechos y objetos reales y materiales no son sino ejemplos imperfectos: de manera que para Platón el conocimiento a priori utiliza afirmaciones que son universales y necesarias, es decir, que valen para todos los casos, que forzosamente tienen que ser así y no pueden ser de otra manera. En otras palabras, dado que el conocimiento es innato al individuo, se trata entonces de un conocimiento a priori, independiente de cualquier tipo de experiencia particular (Montuschi, 2001).

En lo que respecta a la tercera categoría de método científico encontramos el Método hipotético-deductivo. Pensadores como Popper, Hume y Kant apelaban por este método. Mientras los positivistas del círculo de Viena defendían el término de verificación, Popper hablaba de falsación. El concepto de verificación mencionado por Asensi y Parra corresponde a la "acción y el efecto de comprobar si algún enunciado es verdadero o falso. Más específicamente se entiende por 'verificación' el procedimiento adoptado mediante el cual se comprueba la verdad o falsedad de algún enunciado" (2002, p. 8). Contrario a esto, Popper propuso que una teoría podría ser significativa sin necesidad de ser científica, y que la principal característica de la ciencia era concentrarse en afirmaciones falsables, es decir, que pueden probarse como falsas. Popper enfatizó que, independientemente de la cantidad de evidencia que pudiera confirmar una teoría, nunca se puede afirmar al cien por ciento que la hipótesis sea verdadera, e introdujo la noción de corroboración como una medida de cuán bien una hipótesis ha sobrevivido a las pruebas previas, incluso, dio tanta importancia a la falsabilidad, que para él las hipótesis sin falsificadores afirmaban muy poco o nada en absoluto.

Continuando con las categorías de método científico, existe un grupo de filósofos que niegan la existencia de un método como tal. Contrario a Popper, Feyerabend argumentó que no hay ningún método dentro de la historia de la práctica científica que no haya sido violado en algún momento. Este filósofo, considerado por algunos como anarquista y quien abogaba por el "todo vale", rechazaba la idea de un proceder uniforme en la ciencia y pretendía otorgar al investigador la más amplia libertad metodológica. En palabras de Feyerabend:

La idea de un método que contenga principios científicos inalterables y absolutamente obligatorios que rijan los asuntos científicos entra en dificultades al ser confrontada con los resultados de la investigación histórica. En ese momento nos encontramos con que no hay una sola regla por plausible que sea, ni por firmemente basada en la epistemología que venga, que no sea infringida en una ocasión o en otra. Llega a ser evidente que tales infracciones no ocurren accidentalmente, que son el resultado 
de un conocimiento insuficiente o de una falta de atención que pudieron haberse evitado (1993, p. 14).

En otras palabras y tal como lo explica Munévar (2016), la razón por la que a menudo se requiere tal violación, es que el progreso depende a menudo del derrocamiento de la base empírica y la aceptación de nuevas ideas, y ese derrocamiento es en principio posible porque, al determinar la base empírica, hacemos suposiciones teóricas que no necesariamente se postularon siguiendo un método estricto.

\section{Relación filosofía-ciencia}

Por décadas, la relación entre la filosofía y la ciencia ha sido cambiante; dicha relación tiende a ser un poco conflictiva dado que algunos pensadores de la corriente del romanticismo se pueden considerar anticientíficos y, de igual manera, hay ciertas corrientes del lado de la ciencia que podrían considerarse antifilosóficas, como es el caso del positivismo lógico en cabeza de Schlick, Carnap, Feigl, Freud y demás miembros del Ilamado Círculo de Viena. A partir del análisis de esta relación conflictiva, se pretende dar respuesta a las preguntas: ¿se puede filosofar sin ayuda de la ciencia?, ¿puede la ciencia trabajar alejada de la filosofía? o ¿la relación entre la filosofía y ciencia es tan intrínseca que no pueden desarrollarse de manera independiente? Al tratar de responder si se puede filosofar sin ayuda de la ciencia, aflora el pensamiento de que la filosofía no puede, hoy, vivir de espaldas al conocimiento científico (De Azcárraga, 2003). Al respecto, Bunge habla de la "filosofía científica" entendida como:

[...] la clase de concepciones filosóficas que aceptan el método de la ciencia como la manera que nos permite: a) plantear cuestiones fácticas "razonables" (esto es, preguntas que son significativas, no triviales, y que probablemente pueden ser respondidas dentro de una teoría existente o concebible); $y$ b) probar respuestas probables en todos los campos especiales del conocimiento (2014, p. 43).

En el sentido de Bunge, aplicando el método científico, un filósofo estaría en capacidad de adquirir una actitud investigadora para despegarse temporalmente del saber y esforzarse por entender, de manera sistemática, los hechos y situaciones que se presentan ante sus ojos, más allá de la llamada experiencia vital. Con la filosofía científica, todo se resume entonces a qué tan sustentables y confirmables son las ideas del filósofo para que puedan llegar a ser creíbles y lleguen a tener eficacia práctica. Dicho esto, el objeto o meta de este tipo de filosofía es defender la visión científica de la filosofía y que esta debe — como cualquier otra cienciautilizar el método hipotético-deductivo; dicho de otra manera, busca desarrollar, teóricamente, la filosofía para mostrar que la visión científica de esta no solo es capaz de construir teorías sino también de utilizarlas de manera útil en el mundo. 
Este pensamiento de Bunge también llega a conocerse como la filosofía exacta, una filosofía construida con la ayuda de herramientas formales como la lógica y la teoría de conjuntos, cuyas bondades fueron expuestas por Bunge así: "las ventajas de la filosofía exacta son su claridad y su facilitación de la sistematización y la deducción. A su vez, estas características disminuyen los riesgos de interpretaciones textuales sesgadas y de los debates interminables" (2001, p. 193). En definitiva, la filosofía científica se ocupa de problemas generales comunes a todas las ciencias, una filosofía construida por la ciencia y al servicio de la investigación científica.

Por otro lado, la ciencia, al basarse en la observación y la experimentación, podría ser considerada como la fuente de conocimiento más veraz y confiable; no en vano a través de ella se han alcanzado, en los últimos tiempos, desarrollos anteriormente inimaginables. Pero darle entera y completa credibilidad al cientificismo - postura en que la ciencia, y solo la ciencia, describe el mundo como es en sí mismo- , sería practicar una fe ciega y dejar de lado que podríamos estar ante dos caras de una misma moneda. En este punto podríamos afirmar que la filosofía apoya a la ciencia, ya que: i) permite analizar críticamente los presupuestos de la ciencia y cómo estos se articulan con las entidades del mundo; ii) estudia principios, fundamentos, extensión y métodos del conocimiento humano que estimulan el progreso científico — con la llamada epistemología—; y iii) crea un escenario donde los resultados científicos y la experiencia humana se complementan y enriquecen mutuamente.

De hecho, ciertas ciencias están muy ligadas a la filosofía de la mente, como la neurociencia que, siendo un saber más experimental, trata de explicar el sistema nervioso del ser humano, una de las estructuras más complejas del universo, desde diversos puntos de vista, lo cual implica filosofar, no solo por la enorme cantidad de problemas y desafíos aún inexplicables, sino por la necesidad de entender la conducta humana. Luego, no cabe duda de que la neurociencia es un buen ejemplo de que la ciencia no está tan emancipada de la filosofía como puede parecer. Así mismo, existe otra razón por la cual la ciencia necesita a la filosofía y es el hecho de que el conocimiento científico no está separado del resto del conocimiento humano, es decir, los resultados científicos constituyen un conocimiento que puede ser integrado en la búsqueda del ser humano para hallar más respuestas sobre nosotros mismos, sobre el universo y nuestro papel en él. De esta manera, la filosofía ayuda al científico a discutir con otros intelectuales - que no son necesariamente de su campo- y a entender mejor el mundo y a nosotros mismos.

En efecto, tomando como base que la ética es la disciplina filosófica fundada por Aristóteles que estudia el bien y el mal y sus relaciones con la moral y el comportamiento humano, es importante recalcar que en la actualidad esta rama de la filosofía está muy ligada a la ciencia, dado que grandes avances de la tecnología y la ciencia requieren de la ética en temas relacionados con bioética 
como el aborto, la clonación, y demás. De aquí que hoy día se hace especial reconocimiento a personajes como Daniel Bennett, uno de los filósofos de la ciencia más destacados en el ámbito de las ciencias cognitivas, especialmente en el estudio de la conciencia, intencionalidad y la inteligencia artificial.

Sin duda alguna, hasta las mentes más brillantes pueden caer en debates que podrían llegar a considerarse inadmisibles, pero el solo hecho de que este tipo de debates exista resulta muy interesante debido a que permiten que se sigan exponiendo teorías tanto científicas como filosóficas, generando de esa manera la posibilidad de establecer nuevas conexiones entre ambos modelos del pensamiento, tales como las que nacieron con la teoría de la Filosofía de la Ciencia. De hecho, contrario al conflicto que se ha venido generando, debe existir una tendencia que establezca que la relación entre ciencia y filosofía ha de ser de mutua sinergia. La filosofía debe ser importante para la ciencia aun cuando solo se piense en aquella para que permita a los científicos liberar su mente de la necesidad de confiar en el poder de la razón y de la observación experimental. Entonces, la ciencia no puede prescindir de la filosofía, porque hay posturas filosóficas implícitas en las presuposiciones de cualquier paradigma científico y de cómo las distintas teorías científicas podrían estar conectadas a la realidad que ya se conoce.

\section{Conclusiones}

La ciencia, en apoyo a la revolución de la mente humana, ha venido generando una gran cantidad de conocimiento, razón por la cual ha tenido un gran auge el concepto de sociedad del conocimiento. Así mismo, cada día un gran número de personas se suman a la llamada sociedad científica donde cualquier persona puede hacer uso de la ciencia para generar nuevo conocimiento. El hecho de que cada día se perfeccionen las herramientas y los medios para la recolección y análisis de los datos hace que la ciencia en sí se perfeccione constantemente. Sin embargo, el solo deseo de hacer ciencia no es suficiente puesto que, al basarse en suposiciones, la persona que quiere hacer ciencia se enfrenta a un gran número de científicos ya consagrados que buscarán refutar dicha suposición evitando abrirle campo a un nuevo paradigma. Queda en manos del nuevo científico verificar su teoría y abrirse campo y nombre dentro de dicha sociedad.

La filosofía, por su parte, constituye una base fundamental para la ciencia porque es el hecho de cuestionarse lo que hace que un científico mire a su alrededor y busque explicar los fenómenos, insatisfecho por las explicaciones anteriores. Es a través de la filosofía de la ciencia que se deja de considerar a la ciencia como único patrón valido de conocimiento, y se da lugar al cuestionamiento de los paradigmas arraigados hasta el momento, para dar paso a la creación y adopción de nuevos paradigmas como resultado de la aplicación de un método científico. 
A pesar de lo antiguo de la aplicación del método científico, hoy día, científicos de todo el mundo siguen demostrando que es necesaria la implementación de un método para que la ciencia siga desarrollando y realizando aportes a la humanidad. Al no aplicar un método científico se pierde la credibilidad de una investigación y se desvirtúan por completo los "resultados" que cualquier persona que se haga llamar científica quiera dar a conocer.

El hecho de aplicar un método para llegar a la adquisición de conocimiento ha permitido que las publicaciones académicas y científicas estén tan bien estructuradas que garanticen que se realizó un proceso de investigación ordenado y lineal desde la formulación de una pregunta, el diseño de los métodos para responderla, la recopilación de datos, hasta la conclusión del estudio. Sin embargo, algunas publicaciones científicas no especifican el proceso por el cual se produjeron los resultados científicos informados, argumentando que son reconstrucciones que evocan las actividades que se realizaron pero que a menudo no conservan el orden temporal o la lógica de estas actividades. Lo que sí queda totalmente claro es que se requiere, sin lugar a dudas, la aplicación de un método para que la comunidad científica apruebe y adopte los conocimientos que se quieran dar a conocer y, dado el caso, se produzca un cambio de paradigma.

\section{Referencias}

Aristóteles (1995). Tratados de lógica (Órganon) II. Sobre la interpretación. Analíticos primeros. Analíticos segundos. (M. Candel SanMartín, trad.). Madrid: Gredos.

Asensi, V. y Parra. A. (2002). El método científico y la nueva filosofía de la ciencia. Anales de documentación, 5, 9-19.

Bunge, M. (2013). La ciencia, su método y su filosofía. Buenos Aires: Laetoli.

Chávez, G.; Hernández, J.; Chávez, H. y Espinosa, M. (2016). Filosofía de la ciencia de Popper, análisis y reflexiones. Educateconciencia, 11(12), 76-87.

Dávila, G. (2006). El razonamiento inductivo y deductivo dentro del proceso investigativo en ciencias experimentales y sociales. Laurus, 12, 180-205.

Gárnica, E. (2016). La importancia del pensamiento filosófico y científico en la generación de conocimiento. Pensamiento Republicano, (4), 105-114.

Falkenburg, B. (2018). Kant and the scope of the analytic method. Studies in History and Philosophy of Science Part A, 71, 13-23.

Feyerabend, P. (1993). Against Method. London: Third Edition. 
Hassan, N. R. \& Mingers, J. (2018). Reinterpreting the kuhnian paradigm in information systems. Journal of the Association for Information Systems, 19(7), 568-599.

Herbert, J. D. et al. (2000). Science and pseudoscience in the development of eye movement desensitization and reprocessing: Implications for clinical psychology. Clinical Psychology Review, 20(8), 945-971.

Kankam, P. K. (2019). The use of paradigms in information research. Library \& Information Science Research, 41(2), 85-92.

Kant, I. (1998). Critique of Pure Reason. (P. Guyer and A. Wood, trads.). Cambridge: Cambridge University Press.

Kuhn, T. (2002). La estructura de las revoluciones científicas. Buenos Aires: Fondo de Cultura Económica.

Mié, F. (2009). Dialéctica y ciencia en Aristóteles. Revista Signos filosóficos, 11(21), 9-42.

Montuschi, L. (2001). Datos, información y conocimiento. De la sociedad de la información a la sociedad del conocimiento. Serie Documentos de Trabajo de la Universidad del CEMA, 192(6), 2-32.

More, S. J. (2012). Evidence is at the core of scientific method: A challenge for clinicians. The Veterinary Journal, 1(191), 11-12.

Munévar, G. (2016). Historical antecedents to the philosophy of Paul Feyerabend. Studies in History and Philosophy of Science Part A, 57, 9-16.

Popper, K. (1972). Conjeturas y refutaciones. Barcelona: Paidós.

Schwartz, S. A. (2018). Kuhn, Consciousness, and Paradigms. Explore: The Journal of Science and Healing, 14(4), 254-261.

Sale, J. E. \& Thielke, S. (2018). Qualitative research is a fundamental scientific process. Journal of clinical epidemiology, 102, 129-133.

Whewell, W. (1840). The philosophy of the inductive sciences: founded upon their history. Volumen 1. London: JW Parker. 